J3eA - Vol. 2 - 1 (2003).

DOI : 10.1051/bib-j3ea:2003001

\title{
Réflexion sur l'introduction de l'enseignement des microsystèmes dans les différentes filières
}

\author{
I. Dufour *, C. Déjous **, C. Pellet *** et D. Rebière **** (Pôle CNFM de Bordeaux) \\ Mis en ligne le 21 janvier 2003.
}

\begin{abstract}
Résumé
Suite au démarrage de l'industrie des microsystèmes, se pose la question de savoir comment préparer les étudiants à travailler dans ce domaine. En France, différentes filières d'enseignement ont introduit depuis peu des enseignements sur les microsystèmes. Un bilan des différentes formations existant dans le domaine est présenté et l'exemple mené à Bordeaux concernant un enseignement théorique et une initiation à la $\mathrm{CAO}$ (conception assistée par ordinateur) des microsystèmes du premier au troisième cycle est exposé. Nous proposons quelques réflexions concernant l'apport de l'enseignement de cette nouvelle discipline que sont les microsystèmes et présentons les difficultés rencontrées.

Mots-clés : enseignement, microsystèmes.
\end{abstract}

(C) EDP Sciences, 2003.

\begin{abstract}
* Isabelle Dufour ${ }^{1}$, ancienne élève de l'ENS de Cachan, a été reçue à l'agrégation de physique appliquée en 1989 et a obtenu successivement le doctorat en sciences et l'habilitation à diriger des recherches de l'Université Paris-Sud en avril 1993 et en janvier 2000. Depuis décembre 1994, elle occupe un poste de chargée de recherche au CNRS. Depuis septembre 2000, elle effectue son activité de recherche au laboratoire IXL dans l'opération « capteurs microsystèmes ». Son activité de recherche actuelle concerne l'utilisation des microstructures mobiles en tant que microcapteurs chimiques. Elle participe également aux enseignements sur les microsystèmes dispensés à Bordeaux.
\end{abstract}

Adresse postale : Laboratoire IXL, UMR CNRS 5818, Université Bordeaux 1, 351 cours de la Libération F33405 Talence Cedex, France.

e-mail :dufour@ixl.u-bordeaux.fr

** Corinne Déjous ${ }^{1,2}$ a reçu le diplôme d'Ingénieur de l'École Nationale Supérieure d'Électronique, Informatique et Radiocommunications de Bordeaux (ENSEIRB) en 1991. Elle a obtenu le doctorat en sciences en 1994 et est actuellement Maître de Conférences à l'Université Bordeaux 1. Depuis 1991, son activité de recherche se déroule au Laboratoire IXL et concerne les capteurs chimiques à ondes acoustiques.

Adresse postale : Laboratoire IXL, UMR CNRS 5818, Université Bordeaux 1, 351 cours de la Libération, F33405 Talence Cedex, France.

e-mail : dejous@ixl.u-bordeaux.fr

*** Claude Pellet ${ }^{1,3}$, ancien élève de l'ENS de Cachan, occupe depuis 1993 un poste de Professeur des Universités à l'IUT de Bordeaux 1. Son activité de recherche se déroule au Laboratoire IXL au sein de l'opération « capteurs microsystèmes » et concerne l'évaluation d'assemblage, la fiabilité des microsystèmes et la conception de microsystèmes pour des applications médicales.

Adresse postale : Laboratoire IXL, UMR CNRS 5818, Université Bordeaux 1, 351 cours de la Libération F- 
33405 Talence Cedex, France.

e-mail : pellet@ixl.u-bordeaux.fr

**** Dominique Rebière ${ }^{1,3}$ a reçu successivement la Maîtrise EEA, le DEA d'électronique, le doctorat en sciences et l'habilitation à diriger des recherches de l'Université de Bordeaux 1 en 1987, 1988, 1992 et 2001. Il est actuellement Maître de Conférences à l'IUT de Bordeaux 1 dans le Département Mesures Physiques. Depuis 1989, ses activités de recherche se font au laboratoire IXL et concernent les capteurs à ondes acoustiques.

Adresse postale : Laboratoire IXL, UMR CNRS 5818, Université Bordeaux 1, 351 cours de la Libération F33405 Talence Cedex, France.

e-mail : rebiere@ixl.u-bordeaux.fr

\author{
${ }^{1}$ Laboratoire IXL - CNRS UMR 5818 - ENSEIRB / Université Bordeaux 1 \\ ${ }^{2}$ UFR de Physique - Université Bordeaux 1 \\ ${ }^{3}$ IUT Bordeaux 1
}

\title{
1. Introduction
}

Aujourd'hui, vu le nombre de start-ups créées dans le domaine des microsystèmes, on peut estimer que l'on assiste au démarrage de l'industrie des microsystèmes. En effet, ces systèmes miniaturisés constitués de capteurs et/ou d'actionneurs ainsi que de fonctions de traitement sont en train de s'implanter dans de nombreux secteurs industriels (automobile, médical, télécommunications, etc.) et apportent perception et action au côté de l'intelligence des circuits électroniques intégrés. Une question essentielle se pose alors : sachant que les débouchés ne sont pas encore clairement identifiés, faut-il modifier les filières d'enseignement pour présenter ces nouveaux objets que sont les microsystèmes?

Nous verrons, dans la deuxième partie de cet article, que nous sommes relativement nombreux à penser qu'il est important d'introduire l'enseignement des microsystèmes dans les filières d'enseignement. Une fois convaincu qu'il faut parler aux étudiants des microsystèmes, reste à savoir de quelle façon aborder cet enseignement et de nombreuses questions se succèdent alors :

- À quel niveau doit-on introduire les microsystèmes (premier, deuxième et troisième cycle) ?

- Faut-il que ce soit juste une sensibilisation ou un enseignement à part entière ?

- Cela doit-il être un module optionnel ou faire partie d'un tronc commun ?

- Quelles sont les connaissances de base nécessaires à un tel enseignement ?

- La pluridisciplinarité de ces objets les rend-elle attractifs ou au contraire complique-t-elle beaucoup leur compréhension?

- Cet enseignement nécessite-il des moyens lourds ?

- etc.

Dans cet article, nous ne prétendons pas répondre à toutes ces questions mais nous espérons apporter quelques éléments de réflexion en faisant tout d'abord un bilan des formations microsystèmes existant en France puis en présentant notre expérience au niveau des formations de Bordeaux.

\section{Bilan des formations existantes}

\subsection{Origine de ce bilan}

Le Club EEA (Club des Enseignants et des Chercheurs en Électronique, Électrotechnique et Automatique) a organisé les 14 et 15 mars 2002 à Bordeaux des journées sur le thème de « La place des microsystèmes dans l'EEA » (Électronique, Électrotechnique et Automatique). Dans le but de préparer la table ronde sur l'« Enseignement des microsystèmes » qui a clôturé ces journées, une enquête nationale avait été lancée afin 
d'avoir une idée aussi précise que possible de ce qui existait en France. Le questionnaire qui a été diffusé largement par e-mail est présenté figure 1.

\section{Enquête : I'Enseignement des Microsystèmes en France}

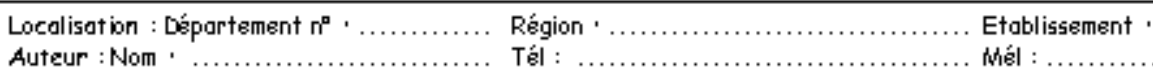

- $y$-a-t-il, dans les formations dans lesquelles vous intervenez un enseignement :

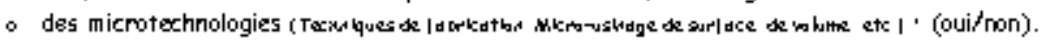

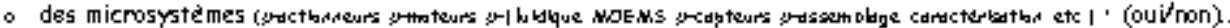

- Si oui, merci d'en indiquer les principales caractéristiquss dans le tableau suivant :

\begin{tabular}{|c|c|c|c|c|c|c|c|c|c|}
\hline \multirow{3}{*}{ 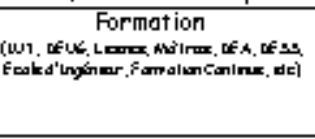 } & \multirow{3}{*}{ 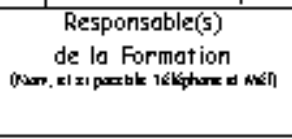 } & \multirow{3}{*}{$\begin{array}{l}\text { Nombre } \\
\text { d'etudiants } \\
\text { concemes }\end{array}$} & \multirow{3}{*}{$\begin{array}{l}\text { Volume } \\
\text { Horaine } \\
\text { Annuel }\end{array}$} & \multicolumn{6}{|c|}{ Répart ition (Heures $/$ Etudiant) } \\
\hline & & & & \multirow[b]{2}{*}{ Gours } & \multirow{2}{*}{ SAdri- } & \multicolumn{3}{|c|}{ Exevgrement Pratique } & \multirow[b]{2}{*}{ Alutrea } \\
\hline & & & & & & $\cos$ & $\begin{array}{c}\text { Futolkd- } \\
\text { that }\end{array}$ & $\begin{array}{l}\text { cordctd- } \\
\text { restbat }\end{array}$ & \\
\hline & & & & & & & & & \\
\hline & & & & & & & & & \\
\hline & & & & & & & & & \\
\hline
\end{tabular}

- Quelle est la date de création de ces enseignements sur les microsystèmes ?

- Quelle est l'origine des intervenants (EA, physique, mécanique, optique, chimie, matériau, biologie)?

- Commentaires ou compléments d'information, en partialier les diffialtés rencontrées :

Áfin de faciliter le traitement, merci de globaliser vos réponses par établissement, et d'envoyer votre document par courrier électronique cobjet ' EEA-MEMS) à : dfouma

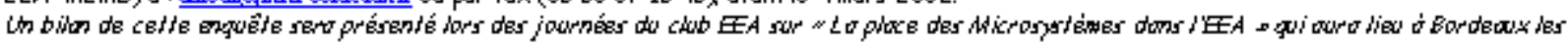
14 et 15 wars 2002 ( woir progrimane of formaloire d'inscription jorits).

Fig. 1. Questionnaire de l'enquête sur l'enseignement des microsystèmes en France.

Nous présentons ici la synthèse de cette enquête. Bien que nous ayons essayé d'avoir une diffusion large du questionnaire, nous ne pouvons prétendre couvrir toutes les formations. Les résultats présentés ne sont donc pas exhaustifs, mais ils permettent de montrer les tendances en termes de progression et de choix concernant ce nouveau type d'enseignement.

La synthèse présentée couvre 10 universités, 4 IUT et 13 écoles d'ingénieurs. Comme nous pouvons le voir sur la figure 2, ces formations sont relativement bien réparties sur tout le territoire français.

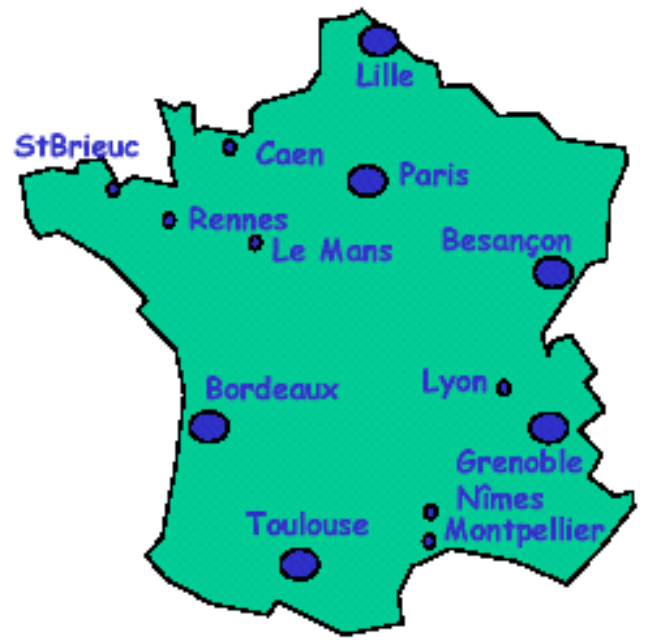

Fig. 2. Répartition géographique des formations microsystèmes en France. 
La liste des différents établissements ayant répondu à l'enquête et le tableau récapitulatif détaillant les différentes réponses au questionnaire est disponible en cliquant ici. Les sites internet des différents établissements ayant répondu à l'enquête sont donnés dans la section 5 (Sites internet).

\subsection{Niveau des formations et flux d'étudiants formés}

Comme le montre le tableau de la figure 3, 41 formations comportant des enseignements sur les microsystèmes (soit un total de 1159 étudiants) ont été identifiées. Ces formations s'étendent du premier au troisième cycle et, suivant les cas, cet enseignement est :

- $\quad$ soit un séminaire de présentation sur les microsystèmes (1 heure 30) ;

- $\quad$ soit un enseignement de base (une quinzaine d'heures) ;

- $\quad$ soit un module complet dédié aux microsystèmes (une cinquantaine d'heures) ;

- $\quad$ soit, de façon plus rare et seulement en troisième année d'école d'ingénieurs, la quasi-totalité d'une année d'enseignement est consacrée aux microsystèmes.

\begin{tabular}{|c|c|c|c|c|}
\hline \multicolumn{3}{|c|}{ Formations } & \multirow{2}{*}{$\begin{array}{c}\text { Nbre } \\
\text { Etudiants }\end{array}$} & \multirow{2}{*}{$\begin{array}{l}\text { Volume } \\
\text { horaire }\end{array}$} \\
\hline Niveau & Type & Nbre & & \\
\hline \multirow[t]{2}{*}{ 1er Cycle } & DEUG & 1 & 150 & 1.5 \\
\hline & Techniciens & 5 & 372 & $8 \rightarrow 31$ \\
\hline \multirow[t]{2}{*}{ 2ème Cycle } & $\begin{array}{l}\text { Maîtr ise. } \\
\text { IUP }\end{array}$ & 3 & 130 & $12 \rightarrow 60$ \\
\hline & $\begin{array}{c}\text { Ingénieur's } \\
\text { (2ème année) }\end{array}$ & 3 & 80 & $17 \rightarrow 60$ \\
\hline \multirow[t]{3}{*}{ 3ème Cycle } & $\begin{array}{c}\text { Ingénieurs } \\
\text { (3ème année) }\end{array}$ & 13 & 240 & $8 \rightarrow 272$ \\
\hline & $\mathrm{DEA}$ & 9 & 118 & $10 \rightarrow 60$ \\
\hline & DESS & 4 & 69 & $21 \rightarrow 30$ \\
\hline Total & & 38 & 1159 & \\
\hline
\end{tabular}

Fig. 3. Niveaux des formations, flux d'étudiants en 2001.

Les figures 4 et 5 montrent bien la rapide progression du nombre de formations incluant un enseignement des microsystèmes à partir de 1998. Cette progression s'est faite à tous les niveaux mais de façon plus lente en premier et deuxième cycle qu'en troisième cycle. Par contre le volume des étudiants concernés diffère fortement suivant s'il s'agit du premier cycle (promotions importantes) ou du troisième cycle (dans le cas des écoles d'ingénieurs cet enseignement ne concerne généralement qu'une option et non pas toute la promotion et dans le cas des DEA il s'agit de petites promotions). De ce fait, suivant le niveau et surtout le nombre d'étudiants concernés, les enseignements des microsystèmes sont abordés de façon différente comme nous allons le voir dans la section 2.3 .

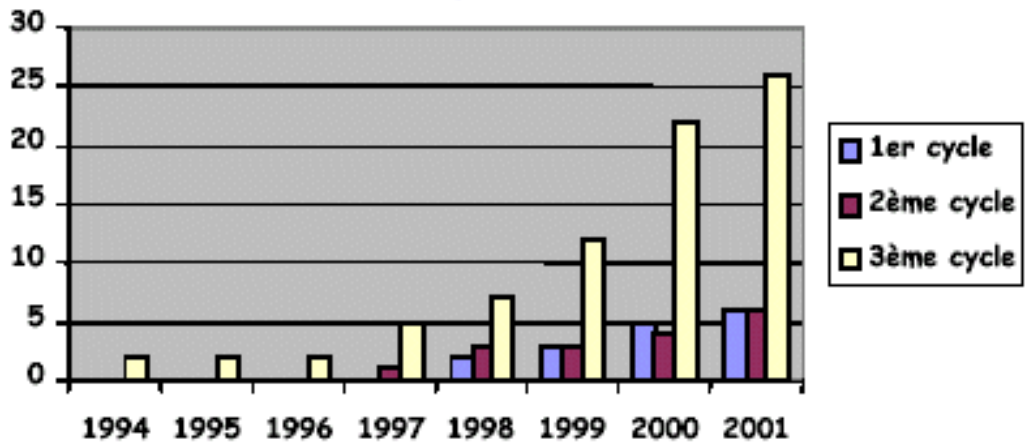

Fig. 4. Évolution du nombre de formations microsystèmes. 


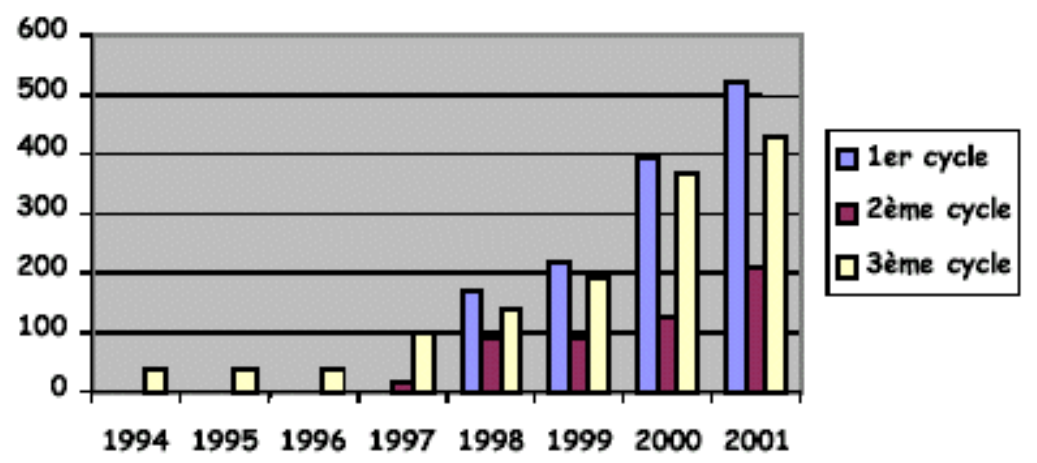

Fig. 5. Évolution du nombre d'étudiants formés aux microsystèmes.

Remarque : quelques cycles de formation continue existent déjà en France mais la proportion de réponses concernant ce type de formation était trop faible pour être exploitée.

\subsection{Contenus des enseignements}

De façon générale, l'enseignement des microsystèmes peut comporter trois types d'interventions :

- cours théoriques éventuellement accompagnés de séminaires ;

- travaux pratiques de simulation des microsystèmes (CAO);

- travaux pratiques de réalisation d'un (ou d'une partie d'un) microsystème, la réalisation du microsystème étant généralement suivie d'une caractérisation de ce dernier.

La figure 6 indique, suivant les niveaux, le nombre de formations ne faisant que des cours théoriques ou incluant la $\mathrm{CAO}$ et/ou la fabrication d'un microsystème.

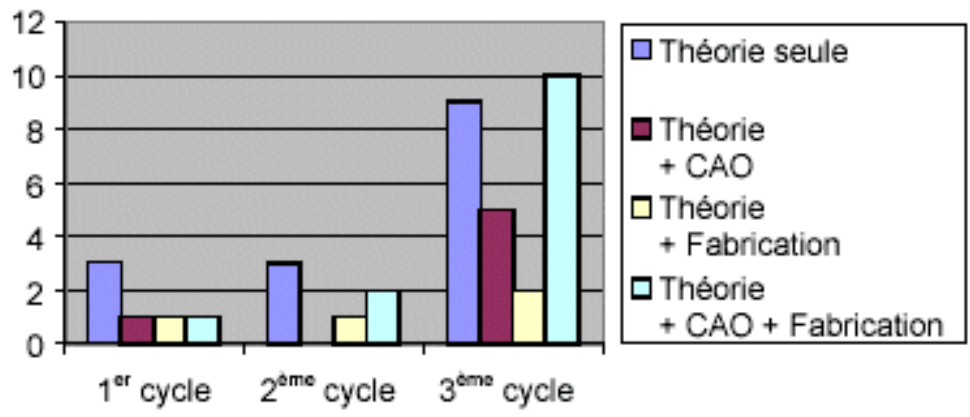

Fig. 6. Répartition des enseignements en 2001.

On peut constater que les parties fabrication ou CAO sont essentiellement abordées en troisième cycle, dans les modules de troisième année d'école d'ingénieurs ou en DEA, et concernent pour chaque formation un petit nombre d'étudiants (de 10 à 30 étudiants). En ce qui concerne les premier et deuxième cycles les enseignements ne comportent en général que des cours théoriques, mises à part quelques rares exceptions.

La figure 7 indique les réponses données au cours de l'enquête concernant l'origine des intervenants (attention il ne s'agit pas de la proportion d'enseignants d'un domaine, ce diagramme indique seulement le nombre de fois où un domaine a été cité dans le coupon réponse de l'enquête). 


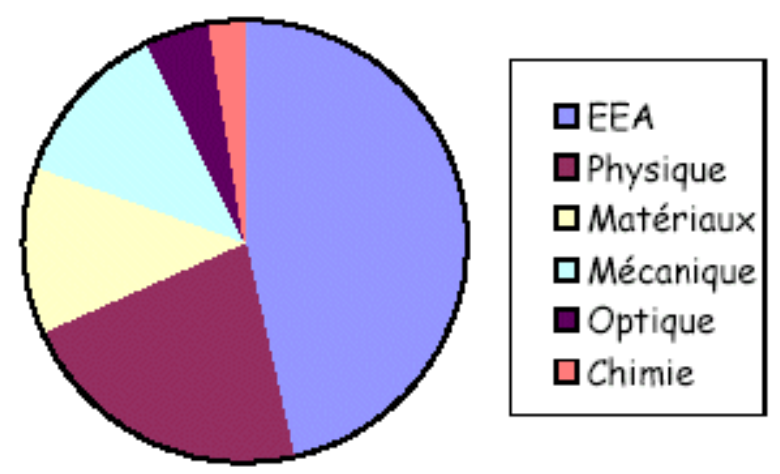

Fig. 7. Origine des intervenants.

On peut constater que pour une grande part, les réponses indiquent que les enseignants sont issus de l'EEA. Ceci est certainement faussé par l'origine de l'enquête (club EEA) mais reflète tout de même la réalité puisque les enseignements des microsystèmes se font essentiellement dans les filières à dominante électronique. Il faut noter l'intervention essentielle des enseignants physiciens, mécaniciens et spécialistes des matériaux. En fait une des composantes nouvelles dans les microsystèmes par rapport à la microélectronique concerne la mécanique et la résistance des matériaux.

\section{L'enseignement des microsystèmes à Bordeaux : du premier au troisième cycle}

\subsection{Formations concernées}

Au niveau du Pôle CNFM (Comité National de Formation en Microélectronique) de Bordeaux, jusqu'à la rentrée 2001/2002 seuls quelques étudiants du DEA d'électronique étaient sensibilisés aux microsystèmes par l'intermédiaire de cours théoriques essentiellement basés sur la présentation et l'étude de microcapteurs physiques et chimiques.

Depuis maintenant un an, l'enseignement des microsystèmes à Bordeaux s'est étendu tant du point de vue du public visé que du contenu. En effet, cet enseignement touche actuellement un peu plus d'une centaine d'étudiants des premier et troisième cycles : 48 étudiants de l'IUT Mesures Physiques, 18 étudiants de troisième année de l'ENSEIRB, 16 étudiants du DESS Microélectronique, 16 étudiants du DESS Qualité et Fiabilité et 6 étudiants du DEA d'Électronique. Comme nous allons le détailler dans la partie suivante, tous ces étudiants suivent des cours théoriques qui diffèrent suivant leur formation et font un stage sur la CAO des microsystèmes.

\subsection{Contenu des enseignements}

Le stage sur la CAO des microsystèmes, qui dure suivant les formations entre un jour et demi et deux jours et demi, est similaire pour toutes les formations du premier au troisième cycle. Seuls les cours théoriques diffèrent assez sensiblement suivant les formations :

- Les étudiants du DEA d'électronique suivent des cours essentiellement basés sur l'étude détaillée des microcapteurs chimiques ( 24 heures) : ceci correspond à un des thèmes de recherche du laboratoire IXL.

- Les étudiants de l'IUT Mesures Physiques sont plutôt sensibilisés aux principes de mesure des microcapteurs physiques (16 heures).

- Pour les autres formations (DESS et troisième année d'école d'ingénieurs), le déroulement des enseignements des microsystèmes se fait typiquement sur une semaine avec $50 \%$ de théorie (cours général) et $50 \%$ de pratique (stage sur la CAO des microsystèmes). 


\subsubsection{Enseignement théorique}

L'enseignement théorique des microsystèmes est fait de façon classique et aborde les différents points suivants :

- Introduction : définition, concept, avantages, marché, domaines d'application, filières de fabrication.

- Procédés de fabrication : spécificités des microsystèmes, techniques de dépôts, de gravure, de moulage, de soudure, problèmes courants.

- Microactionneurs : définition, modes d'actionnement, effet d'échelle, mise en oeuvre, exemples de microactionneurs existants (micropompes, microvalves, micromiroirs, micromoteurs, etc.), principes de modélisation et de simulation, notion de fiabilité des microactionneurs.

- Microcapteurs physiques : définition, accéléromètres, capteurs de pression, de température.

- Microcapteurs chimiques : définition, applications, couches sensibles, capteurs à ondes acoustiques, traitement associés, exemples de réalisation.

- Exemple de fabrication de microdispositifs : détail du procédé de fabrication d'éléments simples et présentation des méthodes de caractérisation.

Alors que les cours pour les étudiants du DEA d'électronique sont focalisés sur les micocapteurs chimiques (24 heures), ceux pour les étudiants de l'IUT couvrent principalement l'étude des microcapteurs physiques (16 heures). Par contre, les cours pour les étudiants des DESS (15 heures) couvrent les différents domaines cités cidessus.

\subsubsection{Stage : CAO des microsystèmes}

Ce stage comporte deux parties. Il met d'abord en oeuvre des outils de conception Memscap sous environnement Cadence puis utilise le logiciel Ansys.

Les outils de conception Memscap viennent se greffer sur l'environnement du logiciel Cadence et introduisent des spécificités liées aux microsystèmes. Les TP concernant l'utilisation des logiciels de Memscap se décomposent en quatre parties :

- Dans un premier temps, les étudiants apprennent à se servir des apports de du logiciel dans la partie Virtuoso de Cadence correspondant au dessin des masques (layout) :

- utilisation de la librairie des composants standards microsystèmes ;

○ utilisation des outils permettant de visualiser une coupe verticale du composant à partir des masques dessinés ;

- utilisation des outils permettant de générer un fichier utilisable sous le logiciel Ansys pour visualiser une vue $3 \mathrm{D}$ du microsystème et faire des simulations par éléments finis ;

- utilisation des outils permettant de dessiner des éléments circulaires, de générer automatiquement des bossages ou trous dans les couches de polysilicium, etc. ;

○ utilisation de tous ces outils pour générer le dessin des masques d'un résonateur (Fig. 8). 


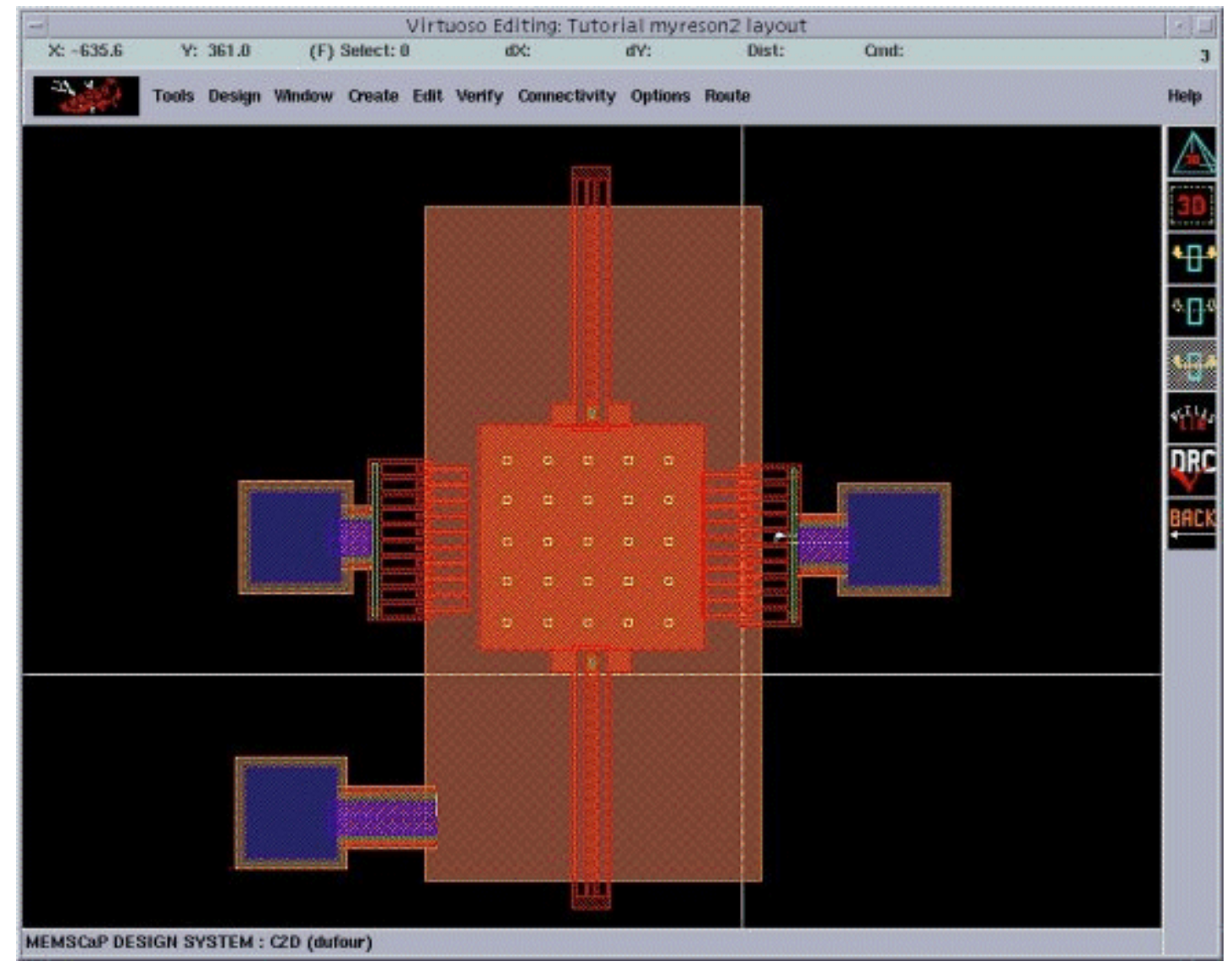

Fig. 8. Dessin des masques d'un résonateur électrostatique (logiciel Memscap).

- Ensuite, les apports du logiciel dans la partie Schematic Composer de Cadence correspondant au dessin du schéma fonctionnel du système (schematics) sont utilisés :

○ utilisation de la librairie de composants standards microsystèmes ;

○ utilisation de cette librairie pour générer le schéma fonctionnel d'un accéléromètre (Fig. 9). 


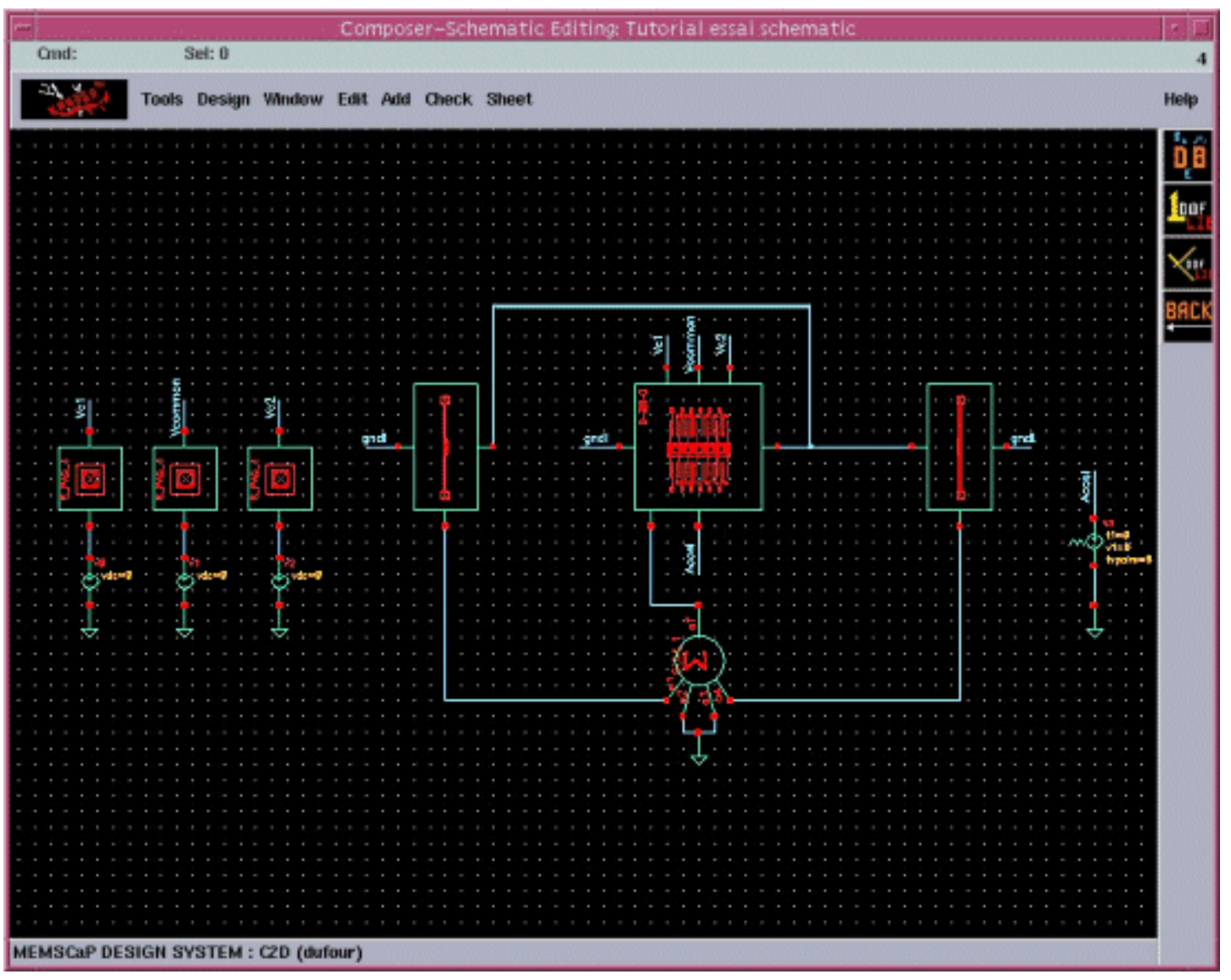

Fig. 9. Schéma fonctionnel d'un accéléromètre.

- La troisième partie du TP est consacrée à la simulation sous Analog Artist. Une simulation comportementale de l'accéléromètre dont le schéma a été créé dans la partie précédente est effectuée (Fig. 10). 


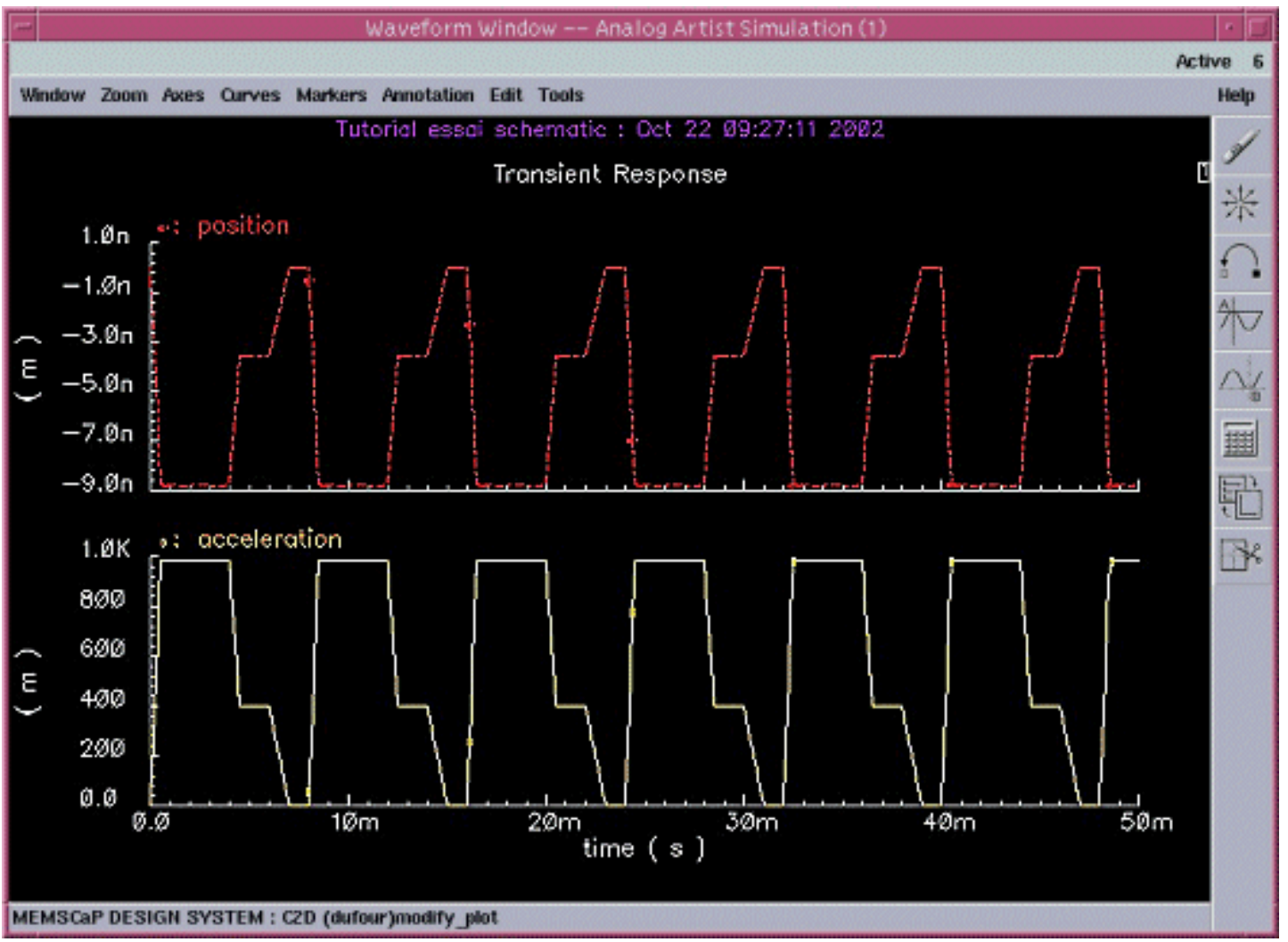

Fig. 10. Visualisation des résultats de la simulation de l'accéléromètre.

- Pour terminer, les étudiants voient comment à partir d'un schéma fonctionnel (schematic) il est possible de générer les dessins des masques (layout).

Le TP concernant l'utilisation du logiciel Ansys a pour but de montrer aux étudiants la démarche à adopter pour effectuer des simulations utilisant la méthode des éléments finis. L'exemple utilisé pour cela est la simulation du comportement statique et dynamique d'une micropoutre en silicium. Chaque simulation se décompose en trois étapes :

- utilisation du préprocesseur pour spécifier les données : géométrie, constantes des matériaux, types d'éléments utilisés pour le maillage, etc. (Fig. 11);

- utilisation du solveur pour la résolution du problème posé après définition du type d'analyse qui va être réalisée, des conditions aux limites, forces, pressions, etc. ;

- utilisation du postprocesseur pour analyser les résultats : visualisation et exploitation des résultats (Fig. 12). 


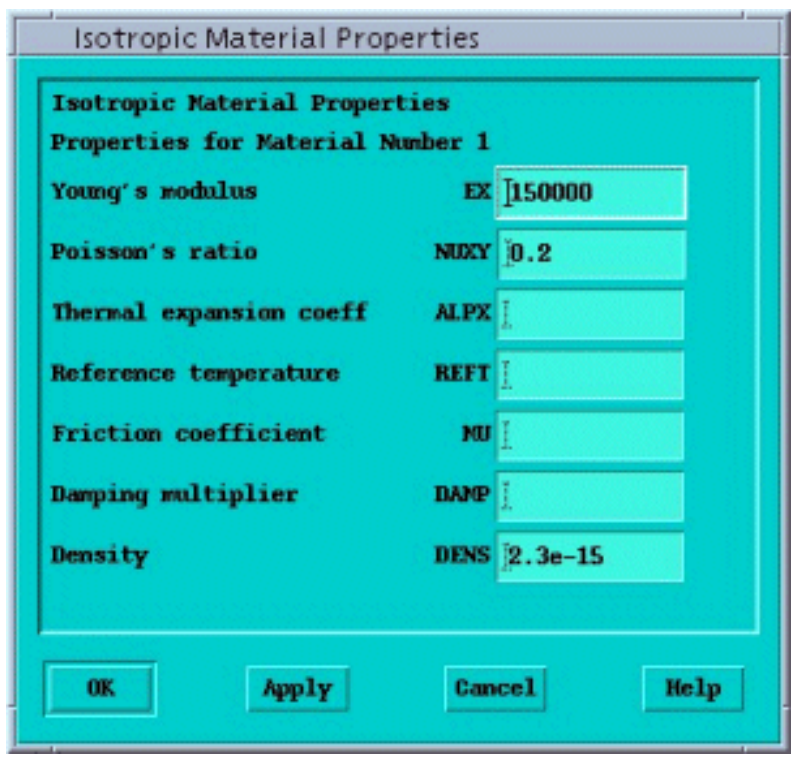

Fig. 11. Saisie des propriétés des matériaux sous le logiciel Ansys.

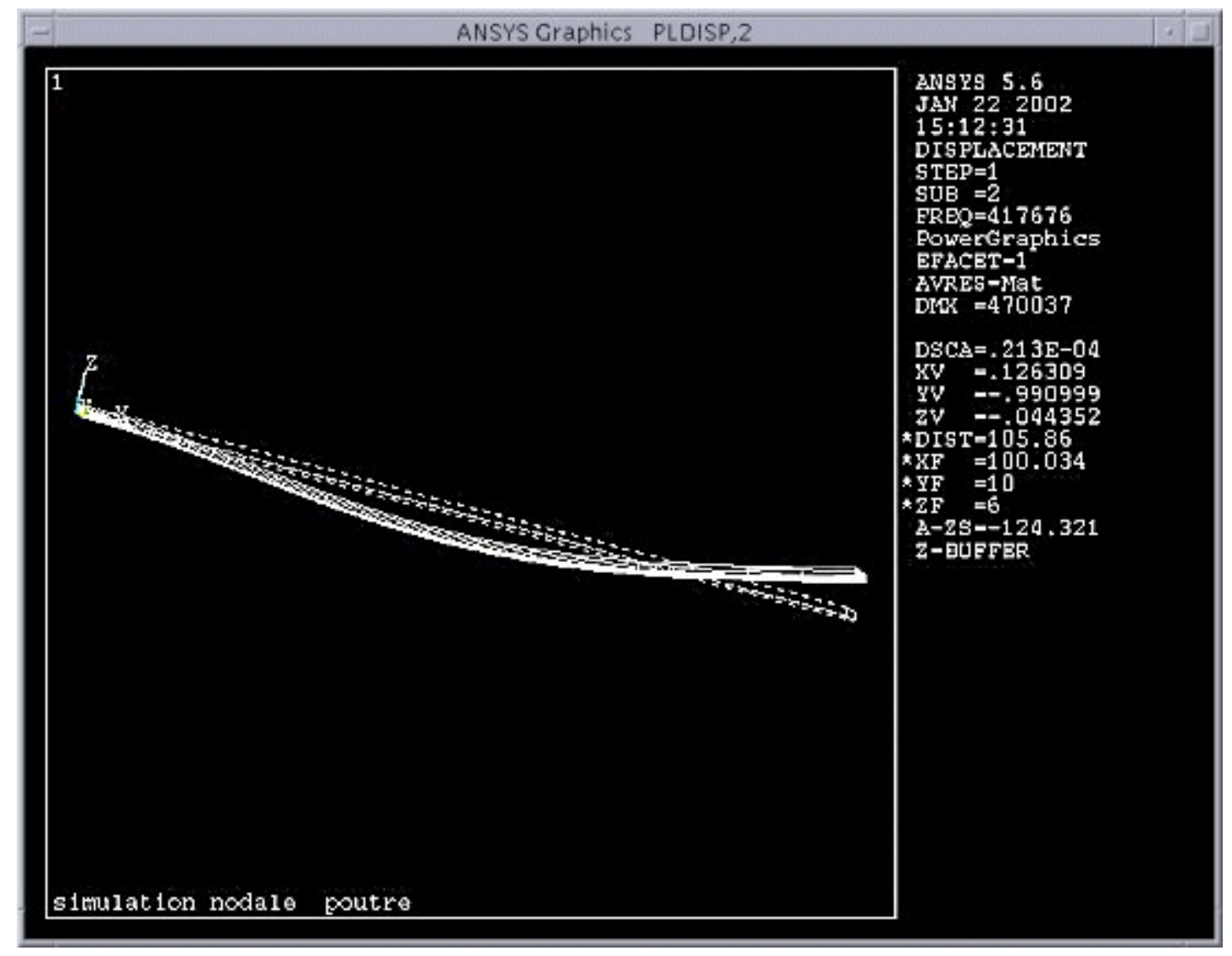

Fig. 12. Visualisation du premier mode de vibration d'une micropoutre. 


\subsection{Bilan}

Au terme de la première année de fonctionnement de cet enseignement des microsystèmes auprès des étudiants de premier et troisième cycles, il apparaît un intérêt certain des étudiants pour « l'objet microsystème ».

La mise en place du stage pratique sur la CAO des microsystèmes au niveau de l'option Technique Instrumentale du département Mesures Physiques de l'IUT était une initiative nouvelle et, afin de savoir comment les étudiants avaient perçu cette formation, nous leur avons proposé de remplir un questionnaire et de noter leurs remarques. Les résultats du questionnaire sont présentés sur la figure 13. En ce qui concerne les remarques, deux idées reviennent souvent et sont résumées par ces deux commentaires d'étudiants :

- «l'étude des microsystèmes est une bonne manière de mettre en application les différentes connaissances acquises au Département »;

- " il serait bien de pouvoir réaliser un microsystème».

Evaluer l'intérêt du sujet traité :

\begin{tabular}{|c|c|c|c|c|}
\hline $\begin{array}{c}\text { Très } \\
\text { intéressant }\end{array}$ & $\begin{array}{c}\text { Assez } \\
\text { intéressant }\end{array}$ & $\begin{array}{c}\text { Moyennement } \\
\text { intéressant }\end{array}$ & $\begin{array}{c}\text { Pas très } \\
\text { intéressant }\end{array}$ & Sans opinion \\
\hline $\mathbf{2 1} \%$ & $\mathbf{7 4} \%$ & $\mathbf{5} \%$ & $\mathbf{0} \%$ & $\mathbf{0} \%$ \\
\hline
\end{tabular}

La durée vous semble-elle :

\begin{tabular}{|c|c|c|c|c|}
\hline Trop longue & Un peu longuc & Adaptée & Un peu courte & Trop courte \\
\hline $\mathbf{3} \%$ & $\mathbf{2 9} \%$ & $\mathbf{4 5} \%$ & $\mathbf{2 4} \%$ & $\mathbf{0} \%$ \\
\hline
\end{tabular}

Le rythme vous semble-t-il :

\begin{tabular}{|c|c|c|c|c|}
\hline Trop rapide & Un peu rapide & Adapté & Un peu lent & Trop lent \\
\hline $\mathbf{2 1} \%$ & $\mathbf{7 4} \%$ & $\mathbf{5} \%$ & $\mathbf{0} \%$ & $\mathbf{0} \%$ \\
\hline
\end{tabular}

Ce module vous semble-t-il globalement:

\begin{tabular}{|c|c|c|c|}
\hline Très utile & Utile & Peu utile & Inutile \\
\hline $\mathbf{5} \%$ & $\mathbf{9 2} \%$ & $\mathbf{3} \%$ & $\mathbf{0} \%$ \\
\hline
\end{tabular}

Ce module a-t-il répondu à vos attentes :

\begin{tabular}{|c|c|c|c|}
\hline Totalement & $\begin{array}{c}\text { Dans } \\
\text { l'ensemble }\end{array}$ & A peu prés & Pas du tout \\
\hline $\mathbf{5} \%$ & $\mathbf{9 7} \%$ & $\mathbf{2 1} \%$ & $\mathbf{0} \%$ \\
\hline
\end{tabular}

Fig. 13. Évaluation par les étudiants de l'IUT des 2,5 jours de formation CAO des microsystèmes.

Durant ces formations, de par la forte pluridisciplinarité des différents microsystèmes évoqués, différents domaines de la physique et de l'EEA sont abordés : l'électrostatique, la résistance des matériaux, la thermique, l'électromagnétisme, l'électronique, etc. Un des intérêts majeurs de l'enseignement des microsystèmes apparait alors ici et a déjà été évoqué dans les commentaires des étudiants : c'est un moyen attractif de faire le lien entre les différentes disciplines classiquement enseignées dans les cursus EEA avec une ouverture forte vers les matériaux et la mécanique. L'objectif de cette pluridisciplinarité n'est pas nécessairement de former des spécialistes de tout, mais des techniciens et cadres capables de travailler avec des collègues ayant des domaines de compétences différents, afin de mener à bien un projet pluridisciplinaire commun.

Concernant l'extension des enseignements des microsystèmes au niveau du Pôle CNFM de Bordeaux, il est envisagé dans les années à venir de développer un stage de caractérisation et de test et, en collaboration avec d'autres centres CNFM, de sensibiliser les étudiants aux techniques de fabrication spécifiques aux microsystèmes en leur faisant réaliser un microsystème de base.

Nous avons détaillé ici l'enseignement des microsystèmes à Bordeaux. Des initiatives menées sur d'autres sites (Fig. 2) peuvent être consultées à partir des sites internet des établissements répertoriés dans la section 5 (Sites internet). 


\section{Conclusion}

En guise de conclusion nous souhaitons donner notre point de vue sur quatre questions :

- But des enseignements sur les microsystèmes ?

Il s'agit de former des techniciens, ingénieurs ou chercheurs à l'ensemble des techniques qui permettent de modéliser, concevoir, réaliser et caractériser les microsystèmes dans le but de faciliter leur adaptation aux nouveaux métiers des entreprises.

- Comment aborder cet enseignement ? Quelles sont les difficultés ?

Il est possible d'aborder cet enseignement de deux façons : l'objet microsystème peut être pris pour présenter les différentes disciplines de la physique (intéressant par exemple en premier cycle) ou inversement pris pour faire la synthèse des différentes disciplines déjà connues. La pluridisciplinarité des microsystèmes les rendent intéressants mais c'est aussi une difficulté à gérer pour l'enseignement. Dans les filières de l'EEA, les lacunes viennent souvent de la mécanique et de la résistance des matériaux. Il faut donc que ces domaines soient dans les pré-requis ou soient intégrés aux enseignements microsystèmes.

- Quels sont les moyens nécessaires ?

Des moyens importants sont indispensables pour la fabrication, la conception et le test des microsystèmes. Ces moyens coûteux ne permettent généralement pas d'accueillir beaucoup d'étudiants simultanément et impliquent donc un fonctionnement des travaux pratiques en petits groupes. Il semble indispensable de fonctionner en réseau pour partager les moyens et l'expérience.

- Quels sont les débouchés ?

Des besoins existent mais sont difficilement quantifiables au niveau des industriels. La solution est donc peut-être d'enseigner les microsystèmes en complément d'une formation par exemple en conception de circuits intégrés.

\section{Sites internet}

Voici la liste des sites internet des établissements ayant des formations incluant des enseignements sur les microsystèmes et ayant répondu à l'enquête lancée par le club EEA en février 2002 :

- École Centrale de Lille (ECL) : http://www.ec-lille.fr

- Institut Supérieur d'Électronique du Nord (ISEN) : http://www.isen.fr/

- Université Paris VI : http://www.upmc.fr/

- Université Paris XI : http://www.u-psud.fr/orsay/index.nsf

- Formation d'Ingénieurs de l'Université Paris Sud Orsay (FIUPSO) : http://www.fiupso.u-psud.fr/

- Groupe ESIEE : http://www.esiee.fr/index.php

- Université de Caen : http://www.unicaen.fr/unicaen/index.html

- Institut des Sciences de la Matière et du Rayonnement (ISMRA) : http://www.ismra.fr/

- IUT de Saint Brieuc : http://www.iutsb.univ-rennes1.fr/

- Université du Maine : http://www.univ-lemans.fr/

- École Nationale Supérieure d'Ingénieurs du Mans (ENSIM) : http://ensim.univ-lemans.fr/

- Université de France-Comté : http://www.univ-fcomte.fr/

- Institut Supérieur d'Ingénieurs de Franche-Comté (ISIFC) : http://sciences.univfcomte.fr/Etudes/Filieres/ISIFC.pdf

- École Nationale Supérieure de Mécanique et des Microtechniques (ENSMM) : http://www.ens2m.fr/

- École Centrale de Lyon (ECL) : http://www.ec-lyon.fr/

- Université Bordeaux 1 : http://www.u-bordeaux1.fr/ub1/

- IUT Bordeaux $1: \mathrm{http}: / /$ www.iuta.u-bordeaux.fr/

- École Nationale Supérieure d'Électronique, Informatique et Radiocommunications de Bordeaux (ENSEIRB) : http://www.enseirb.fr/

- Université Joseph Fourier : http://www.ujf-grenoble.fr/ujf/fr/welcome.phtml 
- École Nationale Supérieure d'Électronique et de Radioélectricité de Grenoble (ENSERG) : http://www.enserg.fr/index3.htm

- École Nationale Supérieure de Physique de Grenoble (ENSPG) : http://www.enspg.inpg.fr/

- Institut des Sciences et Techniques de Grenoble (ISTG) : http://www-istg.ujf-grenoble.fr/

- IUT de Nîmes : http://www.iut-nimes.fr/

- Université de Montpellier II : http://www.univ-montp2.fr/

- Institut National des Sciences Appliquées de Toulouse (INSA) : http://www.insa-tlse.fr/

(adresses vérifiées le 3 janvier 2003) 